\title{
EFFECTIVENESS FOR INFINITE VARIABLE WORDS AND THE DUAL RAMSEY THEOREM
}

\author{
JOSEPH S. MILLER AND REED SOLOMON
}

\begin{abstract}
We examine the Dual Ramsey Theorem and two related combinatorial principles $\mathrm{VW}(k, l)$ and $\mathrm{OVW}(k, l)$ from the perspectives of reverse mathematics and effective mathematics. We give a statement of the Dual Ramsey Theorem for open colorings in second order arithmetic and formalize work of Carlson and Simpson [1] to show that this statement implies $\mathrm{ACA}_{0}$ over $\mathrm{RCA}_{0}$. We show that neither $\mathrm{VW}(2,2)$ nor $\operatorname{OVW}(2,2)$ is provable in $\mathrm{WKL}_{0}$. These results give partial answers to questions posed by Friedman and Simpson [3].
\end{abstract}

\section{INTRODUCTION}

Our goal is to examine several combinatorial theorems from the point of view of effective mathematics and reverse mathematics. In effective mathematics and reverse mathematics one attempts to establish the computability theoretic and proof theoretic strengths, respectively, of the theorems of ordinary mathematics. In this section, we give a brief description of these programs as well as the statements and definitions for the combinatorics we will study.

In effective mathematics, one typically uses the notion of Turing computability to measure the strength of a theorem $T$. The most basic problem is to assume the objects in the hypotheses of $T$ are computable and to ask how complicated, in terms of Turing degree or arithmetic complexity, the objects in the conclusion of the theorem are. For example, consider the statement that every abelian group $G$ has a subgroup $H$ consisting of the elements of finite order. To analyze this statement, we assume that $G$ is a computable group, which means that its domain is a computable subset of $\mathbb{N}$ and its multiplication operator is a computable partial function defined on the domain of $G$. The question of how complicated $H$ can be often breaks into two steps. First, we attempt to use a diagonalization argument to show that $H$ is not always computable. Once we know that $H$ is not always computable, we try to use a coding argument to produce a sharp upper bound on the complexity of $H$. It is not hard to show in this case that $H$ can code the halting problem. That is, there is a computable group $G$ for which the subgroup $H$ consisting of the elements of finite order satisfies $\mathbf{0}^{\prime} \leq_{T} H$. Since the elements of $H$ clearly form a computably enumerable set, we know that $\mathbf{0}^{\prime} \equiv_{T} H$ and therefore that $H$ can be as complicated as its definition allows.

Date: August 8, 2003.

The first author's research is partially supported by an NSF VIGRE Grant at Indiana University. The second author's research is partially supported by an NSF Postdoctoral Fellowship. 
In reverse mathematics, we measure the complexity of the set theoretic axioms required to prove a particular theorem $T$. Because $Z F$ is too powerful to measure subtle differences in proof theoretic strength, we use second order arithmetic, $Z_{2}$, as our set theory. Once $T$ is appropriately stated in $Z_{2}$, we look for the weakest subsystem of $Z_{2}$ which suffices to prove $T$. More formally, we work over a base theory $\mathrm{RCA}_{0}$ which allows comprehension for $\Delta_{1}^{0}$ formulas and we attempt to find a subsystem $S$ extending $\mathrm{RCA}_{0}$ such that $S$ suffices to prove $T$ and such that $\mathrm{RCA}_{0}$ plus $T$ suffices to prove all the axioms in $S$. The most common subsystems $S$ which occur are (in increasing order of strength): $\mathrm{WKL}_{0}$ (which states that every infinite tree of binary strings has an infinite path), $\mathrm{ACA}_{0}$ (which allows comprehension for arithmetic formulas), $\mathrm{ATR}_{0}$ (which allows sets to be formed by transfinite recursion using arithmetic formulas) and $\Pi_{1}^{1}-\mathrm{CA}_{0}$ (which allows comprehension for $\Pi_{1}^{1}$ formulas). In each of these systems, the formulas used for comprehension are allowed to contain parameters.

Not surprisingly, these two approaches are closely connected. For example, if we work with a model $\mathcal{M}$ (called an $\omega$-model) in which the first order part is the standard copy of the natural numbers, then $\mathcal{M}$ is model of $\mathrm{RCA}_{0}$ if and only if the second order part of $\mathcal{M}$ is closed under Turing reducibility and Turing join. Therefore, the least $\omega$-model of $\mathrm{RCA}_{0}$ has the computable sets as its second order part. Similarly, $\mathcal{M}$ is a model of $\mathrm{WKL}_{0}$ if and only if the second order part is closed under Turing reducibility, Turing join and the existence of an infinite path for each infinite subtree of $2^{<\omega}$, and $\mathcal{M}$ is a model of $\mathrm{ACA}_{0}$ if and only if the second order part is closed under Turing reducibility, Turing join and the Turing jump. The least $\omega$-model of $\mathrm{ACA}_{0}$ has the arithmetic sets as its second order part. There is no least $\omega$-model of $\mathrm{WKL}_{0}$, but there is a model in which every set has low degree. (That is, every set $X$ satisfies $X^{\prime}=\mathbf{0}^{\prime}$.)

These characterizations give a useful method for transferring results of effective mathematics into results of reverse mathematics. We illustrate this method with Ramsey's Theorem [5]. For any $A \subseteq \mathbb{N}$, let $[A]^{k}$ denote the set of all $k$ element subsets of $A$. By a partition, we always mean a partition into nonempty disjoint pieces.

Ramsey's Theorem. The following statement holds for all $k, l \geq 1$. For any partition $[\mathbb{N}]^{k}=C_{0} \cup \cdots \cup C_{l-1}$, there is an infinite set $A$ and an $i<l$ such that $[A]^{k} \subseteq C_{i}$.

For a fixed $k$ and $l$, we refer to the statement of Ramsey's Theorem as $\operatorname{RT}(k, l)$. Any infinite set all of whose $k$ element subsets are contained in one of the partitions (such as $A$ ) is called a homogeneous set. Specker [10] gave the first results in effective mathematics concerning Ramsey's Theorem.

Theorem 1.1. There is a computable partition $[\mathbb{N}]^{2}=C_{0} \cup C_{1}$ which has no computable (or even $\Sigma_{1}^{0}$ ) homogeneous set.

Because the computable sets form the second order part of an $\omega$-model of $\mathrm{RCA}_{0}$, the theorem implies that $\mathrm{RCA}_{0}$ does not suffice to prove $\mathrm{RT}(2,2)$. Jockusch [4] extended Theorem 1.1 by proving that a computable partition of $[\mathbb{N}]^{2}$ need not have a $\Sigma_{2}^{0}$ homogeneous set. He proved even more for partitions of $[\mathbb{N}]^{3}$.

Theorem 1.2. There is a computable partition $[\mathbb{N}]^{3}=C_{0} \cup C_{1}$ such that any homogeneous set $A$ satisfies $\mathbf{0}^{\prime} \leq_{T} A$. 
Since there is an $\omega$-model of $\mathrm{WKL}_{0}$ in which all the sets are low, Theorem 1.2 shows that $\mathrm{WKL}_{0}$ does not suffice to prove $\mathrm{RT}(3,2)$. In fact, the theorem says quite a bit more. Because the formulas used in the comprehension scheme for $\mathrm{ACA}_{0}$ are allowed to contain parameters, the subsystem $\mathrm{ACA}_{0}$ is equivalent to one in which the axioms only state comprehension for $\Sigma_{1}^{0}$ formulas. The proof that there are partitions for $\mathrm{RT}(3,2)$ which encode $\mathbf{0}^{\prime}$ also shows that any model of $\mathrm{RT}(3,2)$ and $\mathrm{RCA}_{0}$ must be closed under $\Sigma_{1}^{0}$ comprehension. Therefore, $\mathrm{RT}(3,2)$ suffices to prove $\mathrm{ACA}_{0}$ over $\mathrm{RCA}_{0}$. Since $\mathrm{ACA}_{0}$ can prove RT $(3,2)$, Theorem 1.2 completely classifies $\mathrm{RT}(3,2)$ in terms of reverse mathematics. (For more details, see Section III.7 in [7].)

In this article, we perform a similar (though less comprehensive) analysis for several variations of Ramsey's Theorem which were introduced by Carlson and Simpson [1] and later used by Simpson [6] to study initial segment constructions in degree theory. We begin with a classical statement of the Dual Ramsey Theorem as proved in $[1]$. Let $(\mathbb{N})^{\mathbb{N}}$ be the set of all partitions of $\mathbb{N}$ into infinitely many pieces and let $(\mathbb{N})^{k}$ be the set of all partitions of $\mathbb{N}$ into $k$ pieces. For partitions $X$ and $Y$ of $\mathbb{N}$, we say $Y$ is coarser than $X$ if every partition block in $X$ is contained in a partition block in $Y$. If $X \in(\mathbb{N})^{\mathbb{N}}$, then $(X)^{k}$ is the set of all $Y \in(\mathbb{N})^{k}$ which are coarser than $X$.

Dual Ramsey Theorem. The following statement holds for all $k, l \geq 1$. For any partition $(\mathbb{N})^{k}=C_{0} \cup \cdots \cup C_{l-1}$ in which each set $C_{i}$ is Borel, there exists an $X \in(\mathbb{N})^{\mathbb{N}}$ and an $i<l$ such that $(X)^{k} \subseteq C_{i}$. (We discuss the topology in Section 2.)

Friedman and Simpson [3] asked for an analysis of the Dual Ramsey Theorem in reverse mathematics. The only result to date is from Slaman [8] and states that the Dual Ramsey Theorem is provable in $\Pi_{1}^{1}-\mathrm{CA}_{0}$. In Section 2, we carefully formalize (in $Z_{2}$ ) a version of the Dual Ramsey Theorem in which each $C_{i}$ is required to be open.

Open Dual Ramsey Theorem. For any partition $(\mathbb{N})^{k}=C_{0} \cup \cdots \cup C_{l-1}$ in which each set $C_{i}$ is open, there exists an $X \in(\mathbb{N})^{\mathbb{N}}$ and an $i<l$ such that $(X)^{k} \subseteq C_{i}$.

We let $\operatorname{ODRT}(k, l)$ stand for this theorem with fixed values of $k$ and $l$. Formalizing a proof given by Carlson and Simpson [1], we show that $\operatorname{ODRT}(k+1, l) \operatorname{implies} \operatorname{RT}(k, l)$ over $\mathrm{RCA}_{0}$. From the discussion above, this immediately shows that for $k \geq 3$ and $l \geq 2, \operatorname{ODRT}(k, l)$ is not provable in $\mathrm{WKL}_{0}$. It also shows that for $k \geq 4$ and $l \geq 2$, $\operatorname{ODRT}(k, l)$ implies $\mathrm{ACA}_{0}$.

Friedman and Simpson [3] also asked for an analysis of Thereom 6.3 from Carlson and Simpson [1], a strengthening of one of the crucial lemmas in the proof of the Dual Ramsey Theorem. We state this principal in two different forms and with slightly different terminology than [3]. Let $A$ denote a nonempty finite alphabet and let $\mathrm{Var}=$ $\left\{x_{n} \mid n \in \mathbb{N}\right\}$ be an infinite set of variables which is disjoint from $A$.

Definition 1.3. An infinite variable word is an $\mathbb{N}$-sequence of elements of $A \cup$ Var in which each $x_{i}$ occurs at least once and at most finitely often. A finite variable word is a finite initial segment of an infinite variable word.

Definition 1.4. A finite or infinite variable word is ordered if all occurrences of $x_{i}$ come before the first occurrence of $x_{i+1}$. 
$W\left(x_{i}\right)$ denotes a (finite or infinite) variable word in which the variable $x_{i}$ occurs at least once (although other variables can occur) and place $\left(W\left(x_{i}\right)\right)$ denotes the location of the first occurrence of $x_{i}$ in $W$. For $c \in A, W\left(c / x_{i}\right)$ denotes the word obtained by substituting $c$ into $W$ for all occurrences of the variable $x_{i}$.

For any variable word $W$, a substitution instance of $W$ is a word $V$ of the same length as $W$ in which all occurrences of each variable in $W$ have been substituted by some letter from $A$. We allow different variables to be substituted by different letters from $A$. Frequently, we want to substitute for all the variables in $W$ except for one distinguished variable. We indicate such a situation by saying that $V\left(x_{i}\right)$ is a substitution instance of all the variables in $W$ except $x_{i}$.

For $n \in \mathbb{N}$, we let $W \mid n$ (called the restriction of $W$ to $n$ ) denote the initial segment of $W$ of length $n$. If $x_{i}$ occurs in $W$ and $n=\operatorname{place}\left(W\left(x_{i}\right)\right)$, then $W \mid n$ is the initial segment of $W$ that ends just before the first occurrence of the variable $x_{i}$.

For an infinite variable word $W$, we let $W(A)$ denote the set of all $\alpha \in A^{<\mathbb{N}}$ such that $|\alpha|=\operatorname{place}\left(W\left(x_{i}\right)\right)$ for some $i \in \mathbb{N}$ and $\alpha$ is a substitution instance of $W$ restricted to $|\alpha|$. That is, $W(A)$ contains all substitution instances of initial segments of $W$ formed by cutting off $W$ just before the first occurrence of $x_{i}$ for each $i \in \mathbb{N}$.

Given these definitions, we let $\operatorname{VW}(k, l)$ be the statement that if $|A|=k$ and $c$ : $A^{<\mathbb{N}} \rightarrow l$ is an $l$-coloring of $A^{<\mathbb{N}}$, then there is an infinite variable word $W$ such that every element of $W(A)$ has the same color. (In this situation, we say that $W$ is a homogeneous word for $c$.) We let $\operatorname{OVW}(k, l)$ be the same statement except that we require $W$ to be an infinite ordered variable word. There is a proof of OVW $(k, l)$ for all $k$ and $l$ in Carlson and Simpson [1, Theorem 6.3]. Friedman and Simpson [3] asked for an analysis of OVW $(k, l)$ and in particular, they asked if this statement is effectively true. In Section 3, we show that neither $\operatorname{OVW}(2,2)$ nor $\mathrm{VW}(2,2)$ is provable in $\mathrm{WKL}_{0}$ and therefore neither is effectively true.

In the last section, we list several open questions.

Our notation is standard. It follows [9] for computability theory, [7] for reverse mathematics and [1] for the Dual Ramsey Theorem. The reader who is interested in more background is referred to [2] for effective mathematics and [7] for reverse mathematics.

\section{Open Dual Ramsey Theorem}

We begin with the definitions necessary to state the Open Dual Ramsey Theorem in $\mathrm{RCA}_{0}$. Throughout, we let $k \in \mathbb{N}$ stand for both the number $k$ and the set $\{0, \ldots, k-1\}$.

Definition 2.1. $\left(\mathrm{RCA}_{0}\right)$ For any $k \geq 1$, a partition of $\mathbb{N}$ into $k$ blocks is a function $f$ from $\mathbb{N}$ onto $k$ such that $f(0)=0$ and for all $0<i<k$ and all $n \in \mathbb{N}$, if $f(n)=i$, then there is an $m<n$ such that $f(m)=i-1$.

Such a function $f$ partitions $\mathbb{N}$ into the blocks $K_{i}=\{n \in \mathbb{N} \mid f(n)=i\}$, for $0 \leq i<k$. Notice that this definition guarantees that if $f$ and $g$ are distinct functions which partition $\mathbb{N}$ into $k$ blocks, then these partitions are different in the sense that neither can be obtained from the other by a permutation of the index labels on the blocks. 
We can represent the class of all such functions as an open set in $k^{\mathbb{N}}$. Define $P_{k}$ to be the set of all strings $\sigma \in k^{<\mathbb{N}}$ such that

(1) $\sigma(0)=0$

(2) $\forall i<k \exists n<|\sigma|(\sigma(n)=i)$,

(3) $\forall i<(k-1) \forall n<|\sigma|(\sigma(n)=i+1 \rightarrow \exists m<n(\sigma(m)=i))$.

Because this definition uses only bounded quantification, $\mathrm{RCA}_{0}$ suffices to prove the existence of $P_{k}$.

For any $\sigma \in k^{<\mathbb{N}}$, we let $[\sigma]$ denote the set of all functions $f: \mathbb{N} \rightarrow k$ such that $\sigma \subset f$. (We do not put any requirements here that $f$ be onto $k$.) For any set $P \subset k^{<\mathbb{N}}$, we let $\mathcal{O}(P)$ denote the union of $[\sigma]$ for $\sigma \in P$. Recall that in the standard product topology on $k^{\mathbb{N}}$ (where $k$ is given the discrete topology), the sets $[\sigma]$ for $\sigma \in k^{<\mathbb{N}}$ form a basis of clopen sets. Therefore, for any set $P \subset k^{<\mathbb{N}}, \mathcal{O}(P)$ is a union of basic open sets and hence $\mathcal{O}(P)$ is open. Our notation $\mathcal{O}(P)$ is intended to indicate that $\mathcal{O}(P)$ is the open set generated by the basic open sets represented in $P$. Later, we will also deal with closed sets generated as the set of paths through a tree. A tree is an nonempty set $T \subseteq k^{<\mathbb{N}}$ (or $T \subseteq \mathbb{N}^{<\mathbb{N}}$ ) such that if $\sigma \in T$ and $\tau \subset \sigma$, then $\tau \in T$. Let $[T]$ denote the set of infinite paths through $T$ and recall that $[T]$ is a closed subset of $k^{\mathbb{N}}$.

Classically, it is clear that the class of all partitions of $\mathbb{N}$ into exactly $k$ pieces is equal to $\mathcal{O}\left(P_{k}\right)$. By the comments above, $\mathcal{O}\left(P_{k}\right)$ is an open set in $k^{\mathbb{N}}$. Furthermore, $\mathrm{RCA}_{0}$ is strong enough to prove that $\mathcal{O}\left(P_{k}\right)$ is not empty. For example, fix any $\sigma \in P_{k}$ and let $f$ be the function such that $\sigma \subset f$ and for all $x \geq|\sigma|, f(x)=0$. Therefore, we use $\mathcal{O}\left(P_{k}\right)$ to represent the class of all partitions of $\mathbb{N}$ into $k$ blocks in $\mathrm{RCA}_{0}$.

We will also need to consider the class of all partitions of $\mathbb{N}$ into an arbitrary, possibly infinite, number of blocks as well as the class of all partitions of $\mathbb{N}$ into infinitely many blocks. For this purpose, we define $\mathcal{T}$ to be the set of all strings $\sigma \in \mathbb{N}<\mathbb{N}$ such that

(1) $\sigma(0)=0$,

(2) $\forall i \forall n<|\sigma|(\sigma(n)=i+1 \rightarrow \exists m<n(\sigma(m)=i))$.

Notice that $\mathcal{T}$ is a tree with no dead ends and that for any $\sigma \in \mathcal{T}$ and any $n<|\sigma|$, $\sigma(n) \leq n$. Therefore, $\mathcal{T}$ is a recursively branching tree. Classically, $[\mathcal{T}]$ contains all the partitions of $\mathbb{N}$ into either finitely or infinitely many blocks. Formally, a block of a partition $f \in[\mathcal{T}]$ is a set of the form $\{x \in \mathbb{N} \mid f(x)=i\}$, where $i$ is an element of the range of $f . \mathrm{RCA}_{0}$ suffices to prove the existence of $\mathcal{T}$ and to prove that $[\mathcal{T}]$ is not empty. For example, the identity function $f(n)=n$ is an element of $[\mathcal{T}]$.

If $f \in[\mathcal{T}]$ and range $(f)=\mathbb{N}$, then $f$ represents a partition of $\mathbb{N}$ into infinitely many blocks. A refinement of $f$ is another partition which possibly collapses some of the partitioned blocks of $f$ into larger blocks, but does not break up any of the blocks of $f$. Formally, $g \in[\mathcal{T}]$ is a refinement of $f$ if $\forall i, j(f(i)=f(j) \rightarrow g(i)=g(j))$. For $f \in[\mathcal{T}]$, let $(f)^{\leq k}$ denote the class of all refinements of $f$ into at most $k$ blocks.

In order to represent $(f)^{\leq k}$, we define the tree $\mathcal{T}(f, k)$ to be the set of all strings $\sigma \in \mathcal{T}$ such that range $(\sigma) \subset k$ and $\forall i, j<|\sigma|(f(i)=f(j) \rightarrow \sigma(i)=\sigma(j))$. $\mathrm{RCA}_{0}$ suffices to prove the existence of $\mathcal{T}(f, k)$ from the parameters $f$ and $k$. Furthermore, for any given $k$, $\mathrm{RCA}_{0}$ can prove that if range $(f)=\mathbb{N}$, then $[\mathcal{T}(f, k)]$ is nonempty since $\mathrm{RCA}_{0}$ can form the path $g_{k}$ such that $g_{k}(i)=f(i)$ if $f(i)<k$ and $g_{k}(i)=0$ otherwise. $g_{k}$ collapses 
all blocks after the $k^{\text {th }}$ block into the first block, and leaves the blocks corresponding to $f(n)=1, \ldots, f(n)=k-1$ unchanged. Since classically, $(f) \leq k$ is exactly $[\mathcal{T}(f, k)]$, we use $[\mathcal{T}(f, k)]$ to represent the class of all refinements of $f$ into at most $k$ blocks in $\mathrm{RCA}_{0}$.

An open l-coloring of $\mathcal{O}\left(P_{k}\right)$ is given by sets $S_{0}, \ldots, S_{l-1} \subset P_{k}$ such that

(1) $\mathcal{O}\left(P_{k}\right)=\mathcal{O}\left(S_{0}\right) \cup \cdots \cup \mathcal{O}\left(S_{l-1}\right)$,

(2) for any $i \neq j, S_{i} \cap S_{j}=\emptyset$,

(3) if $\sigma \in S_{i}$, then $\tau \in S_{i}$ for all $\sigma \subset \tau$ where $\tau \in P_{k}$.

Notice that the sets $\mathcal{O}\left(S_{i}\right)$ are pairwise disjoint by Condition (3). Therefore, the sets $\mathcal{O}\left(S_{i}\right)$ form a disjoint open cover of $\mathcal{O}\left(P_{k}\right)$, which we view as a coloring of $\mathcal{O}\left(P_{k}\right)$.

We can now state the Open Dual Ramsey Theorem for $k$ partitions and $l$ colors in $\mathrm{RCA}_{0}$. For fixed $k$ and $l$, we denote this statement by $\operatorname{ODRT}(k, l)$.

Theorem 2.2. Let $S_{0}, \ldots, S_{l-1}$ be an open coloring of $\mathcal{O}\left(P_{k}\right)$. Then there is a partition $f \in[\mathcal{T}]$ and an $i<l$ such that range $(f)=\mathbb{N}$ and for all partitions $\alpha \in \mathcal{O}\left(P_{k}\right)$, if $\alpha \in[\mathcal{T}(f, k)]$, then $\alpha \in \mathcal{O}\left(S_{i}\right)$.

Before proceeding, we should note that, classically, this theorem is a direct consequence of the Dual Ramsey Theorem. As mentioned above, our coloring consists of open (and hence Borel) sets. Thus, the classical Dual Ramsey Theorem implies that there is a partition $f$ of $\mathbb{N}$ into infinitely many pieces such that all refinements of $f$ into exactly $k$ blocks have the same color. Any $\alpha \in[\mathcal{T}(f, k)]$ is a partition of $\mathbb{N}$ into at most $k$ blocks. But, if this $\alpha$ is in $\mathcal{O}\left(P_{k}\right)$, then $\alpha$ must actually be a partition of $\mathbb{N}$ into exactly $k$ pieces. Therefore, this statement of the Open Dual Ramsey Theorem is a direct consequence of the Dual Ramsey Theorem.

For any string $\sigma \in \mathcal{T}$ or partition $f \in[\mathcal{T}]$, we define

$$
\begin{gathered}
B_{\sigma}=\{i|0<i<| \sigma \mid \wedge \forall j<i(\sigma(j)<\sigma(i))\} \\
B_{f}=\{i \mid 0<i \wedge \forall j<i(f(j)<f(i))\} .
\end{gathered}
$$

In words, $B_{f}$ represents the $\leq_{\mathbb{N}}$-least elements of the blocks of $f$, after the first block. We can now give the main theorem of this section. Both the statement and the proof of this theorem are formalizations in $\mathrm{RCA}_{0}$ of Theorem 3.1 from [1].

Theorem 2.3. $\left(R C A_{0}\right)$ ODRT $(k+1, l)$ implies $R T(k, l)$.

Proof. Recall that $\mathrm{RT}(k, l)$ says that for any $l$-coloring of the $k$-element subsets of $\mathbb{N}$, there is an infinite set $X$ such that all $k$-element subsets of $X$ have the same color. Therefore, we fix a coloring of the $k$-elements subsets of $\mathbb{N}$ written as a partition of the $k$-element subsets of $\mathbb{N}$ into $l$ pairwise disjoint nonempty pieces $C_{0}, \ldots, C_{l-1}$. Define the sets $U_{i}$ for $i<l$ by

$$
U_{i}=\left\{\sigma \in P_{k+1} \mid B_{\sigma} \in C_{i}\right\} .
$$

By the definition of $B_{\sigma}$, it is clear that if $\sigma \in U_{i}$ and $\sigma \subset \tau$ for any $\tau \in P_{k+1}$, then $\tau \in U_{i}$. It is also clear that the $U_{0}, \ldots, U_{l-1}$ are disjoint and $\mathcal{O}\left(U_{0}\right) \cup \cdots \cup \mathcal{O}\left(U_{l-1}\right)=\mathcal{O}\left(P_{k+1}\right)$. Therefore, we can apply the Open Dual Ramsey Theorem to the coloring of $\mathcal{O}\left(P_{k+1}\right)$ given by $U_{0}, \ldots, U_{l-1}$. 
Fix $f \in[\mathcal{T}]$ and $i<l$ as in the statement of the Open Dual Ramsey Theorem. Consider $B_{f}$. Since range $(f)=\mathbb{N}$, we have that $B_{f}$ is infinite. We claim that the set of $k$-element subsets of $B_{f}$ is contained in $C_{i}$, and hence $B_{f}$ is the required infinite homogeneous set.

Fix $\left\{y_{1}, \ldots, y_{k}\right\} \subset B_{f}$ and without loss of generality assume $y_{1}<\cdots<y_{k}$. We will show that $\left\{y_{1}, \ldots, y_{k}\right\} \in C_{i}$. First, we construct $\alpha \in \mathcal{O}\left(P_{k+1}\right)$ such that $\alpha \in$ $[\mathcal{T}(f, k+1)]$ and $B_{\alpha}=\left\{y_{1}, \ldots, y_{k}\right\}$. Fix $n_{i}$ such that $f\left(y_{i}\right)=n_{i}$. We define $\alpha(x)=0$ if $f(x) \notin\left\{n_{1}, \ldots, n_{k}\right\}$ and $\alpha(x)=i$ if $f(x)=n_{i}$. Thus, we have collapsed all blocks in $f$ other than the blocks containing one of the $y_{i}$ elements into a single block in $\alpha$, and we have left each block in $f$ containing one of the $y_{i}$ elements unchanged in $\alpha$. Therefore, $\alpha$ has exactly $k+1$ blocks and $B_{\alpha}=\left\{y_{1}, \ldots, y_{k}\right\}$.

By our choice of $f$, we know $\alpha \in \mathcal{O}\left(U_{i}\right)$. Therefore, if we let $\sigma \subset \alpha$ be an initial segment such that $\sigma$ maps onto $k+1$, then we must have $B_{\sigma}=\left\{y_{0}, \ldots, y_{k-1}\right\}$ and also $B_{\sigma} \in C_{i}$. Therefore, $\left\{y_{0}, \ldots, y_{k-1}\right\} \in C_{i}$ as required.

Corollary 2.4. For any $k \geq 3$ and $l \geq 2, O D R T(k, l)$ is not provable in $W K L_{0}$.

Proof. This follows from Theorem 2.3 and the fact that for $k \geq 2$ and $l \geq 2, \operatorname{RT}(k, l)$ is not provable in $\mathrm{WKL}_{0}$.

Corollary 2.5. (RCA $\left.A_{0}\right)$ For any $k \geq 4$ and any $l \geq 2$, ODRT $(k, l)$ implies $A C A_{0}$.

Proof. This follows from Theorem 2.3 and the fact that for $k \geq 3$ and $l \geq 2, \operatorname{RT}(k, l)$ implies $\mathrm{ACA}_{0}$.

\section{INFINITE VARIABLE WORDS}

In this section, we work with the finite alphabet $A=\{a, b\}$. We begin with the statement of our main theorem.

Theorem 3.1. Let $A=\{a, b\}$. There is a computable two-coloring $c: A^{<\mathbb{N}} \rightarrow 2$ such that $W(A)$ is not homogeneous for any $\Delta_{2}^{0}$ infinite ordered variable word $W$.

Corollary 3.2. $W K L_{0}$ does not suffice to prove $O V W(2,2)$.

Proof. By Theorem 3.1, any model of OVW $(2,2)$ which contains the computable sets must contain non-low sets. However, there is an $\omega$-model of $\mathrm{WKL}_{0}$ containing all the computable sets in which every set is low. (See Corollary VIII.2.18 in [7].)

To connect this statement to $\mathrm{VW}(2,2)$, we use the following simple lemma, which holds for any nonempty alphabet $A$.

Lemma 3.3. Let $A$ be a finite nonempty alphabet. If $W$ is an infinite variable word such that $W(A)$ is homogeneous for the coloring $c$, then there exists an infinite ordered variable word $V$ such that $V(A)$ is homogeneous for $c$ and $V \leq_{T} W^{\prime}$. (W' denotes the Turing jump of $W$.)

Proof. We form $V$ by choosing a subsequence $\left\{y_{i}\right\}_{i \in \mathbb{N}}$ of the variables $\left\{x_{m}\right\}_{m \in \mathbb{N}}$. Let $y_{0}=x_{0}$. Assume that $y_{i}$ has been determined to be $x_{k_{i}}$. Using $W^{\prime}$ we can find the last occurrence of $x_{k_{i}}$ in $W$. Let $x_{k_{i+1}}$ be the first variable which has its first occurrence in 
$W$ after the last occurrence of $x_{k_{i}}$. Set $y_{i+1}=x_{k_{i+1}}$. This defines the sequence $\left\{y_{i}\right\}$ of variables. Let $V$ be the word obtained from $W$ by substituting an arbitrary letter $a \in A$ for each variable $x_{j} \notin\left\{y_{i}\right\}$ and by substituting $x_{i}$ for $y_{i}$. It is clear that $V$ is an infinite ordered variable word and that $V(A)$ is a subset of $W(A)$. Therefore, $V(A)$ is homogeneous for $c$.

Corollary 3.4. $W K L_{0}$ does not suffice to prove $V W(2,2)$.

Proof. By Theorem 3.1 and Lemma 3.3, any $\omega$-model of $\mathrm{VW}(2,2)$ which contains the computable sets must contain a non-low set. That is, suppose $c$ is a computable coloring as in Theorem 3.1 and $W$ is an infinite variable word with low Turing degree for which $W(A)$ is homogeneous. By Lemma 3.3, there is an infinite ordered variable word $V$ which is computable in the Turing jump of $W$ (and hence is $\Delta_{2}^{0}$ ) for which $V(A)$ is homogeneous. This contradicts the choice of $c$.

We begin the proof of Theorem 3.1 with a series of definitions and technical lemmas needed to show that our eventual construction succeeds.

Definition 3.5. A finite set $W_{1}\left(x_{i_{1}}\right), \ldots, W_{n}\left(x_{i_{n}}\right)$ of finite variable words with distinguished variables is admissible if for all $j \neq k \leq n$, place $\left(W_{j}\left(x_{i_{j}}\right)\right) \neq \operatorname{place}\left(W_{k}\left(x_{i_{k}}\right)\right)$.

Definition 3.6. Let $s \in \mathbb{N}$ and let $W_{1}\left(x_{i_{1}}\right), \ldots, W_{n}\left(x_{i_{n}}\right)$ be an admissible set of words such that for all $m \leq n,\left|W_{m}\right|<s$. Let $k_{m}$ be such that $\left|W_{m}\right|+k_{m}=s$. The (undirected and labeled) graph $G$ induced by $W_{1}\left(x_{i_{1}}\right), \ldots, W_{n}\left(x_{i_{n}}\right)$ on $A^{s}$ is defined as follows. The nodes of $G$ are the elements of $A^{s}$, the set of words of length $s$ over $A$. If $\alpha, \beta \in A^{s}$, then we put an edge labeled by $W_{m}$ between $\alpha$ and $\beta$ if and only if there is a substitution instance $\widehat{W}_{m}\left(x_{i_{m}}\right)$ of all the variables in $W_{m}$ except $x_{i_{m}}$ and a string $\delta \in A^{k_{m}}$ such that either

$$
\begin{gathered}
\left(\alpha=\widehat{W}_{m}\left(a / x_{i_{m}}\right) * \delta \text { and } \beta=\widehat{W}_{m}\left(b / x_{i_{m}}\right) * \delta\right) \text { or } \\
\left(\alpha=\widehat{W}_{m}\left(b / x_{i_{m}}\right) * \delta \text { and } \beta=\widehat{W}_{m}\left(a / x_{i_{m}}\right) * \delta\right) .
\end{gathered}
$$

Whenever we use the term "induced graph," we assume that it has been induced by an admissible set of words as described above. Notice that since the variable $x_{i_{j}}$ is assumed to occur in the word $W_{j}$, there cannot be a node $\alpha$ with an edge labeled by $W_{j}$ from $\alpha$ to itself. Furthermore, by the following lemma, dropping the edge labels of an induced graph $G$ results in an ordinary undirected graph without multiple edges between nodes. This property plays no role in our construction, but it is included to give the reader some intuition about these graph structures.

Lemma 3.7. Let $G$ be an induced graph on $A^{s}$. For any pair of distinct elements $\alpha, \beta \in A^{s}$, there is at most one labeled edge between $\alpha$ and $\beta$.

Proof. Suppose not and fix $\alpha, \beta$ and $j \neq k$ such that there are edges labeled by $W_{j}$ and $W_{k}$ between $\alpha$ and $\beta$. Since place $\left(W_{j}\left(x_{i_{j}}\right)\right) \neq \operatorname{place}\left(W_{k}\left(x_{i_{k}}\right)\right)$, we can assume without loss of generality that place $\left(W_{j}\left(x_{i_{j}}\right)\right)<\operatorname{place}\left(W_{k}\left(x_{i_{k}}\right)\right)$. The fact that $\alpha$ and $\beta$ differ at the location place $\left(W_{j}\left(x_{i_{j}}\right)\right)$ contradicts the existence of an edge labeled by $W_{k}$ between $\alpha$ and $\beta$. 
Lemma 3.8. Let $G$ be an induced graph on $A^{s}$. G has only even length cycles.

Proof. Fix any cycle in $G$ and a node $\alpha$ in this cycle. Assume that the labels that occur in the cycle are $W_{1}, \ldots, W_{n}$ and that $\operatorname{place}\left(W_{1}\left(x_{i_{1}}\right)\right)<\cdots<\operatorname{place}\left(W_{n}\left(x_{i_{n}}\right)\right)$. It suffices to show by induction that each of these labels occurs on an even number of edges in the cycle.

Consider the label $W_{1}$. Imagine starting at $\alpha$, traveling around the cycle exactly once and keeping track of the letter which occurs in position place $\left(W_{1}\left(x_{i_{1}}\right)\right)$. Since place $\left(W_{1}\left(x_{i_{1}}\right)\right)$ is the least among the places of the distinguished variables of the labels in this cycle, the letter in this position can change only when we cross an edge labeled by $W_{1}$. Since the letter in place $\left(W_{1}\left(x_{i_{1}}\right)\right)$ must have its original value when we return to $\alpha$ after going around the cycle, there must be an even number of edges labeled by $W_{1}$.

Assume by induction that each label $W_{i}$ for $i<m$ occurs an even number of times in the cycle and consider the label $W_{m}$. As before, imagine starting at $\alpha$, traveling once around the cycle and keeping track of the letter which occurs in position place $\left(W_{m}\left(x_{i_{m}}\right)\right)$. The letter in this position will change every time we cross an edge labeled $W_{m}$, the letter cannot change when we cross an edge labeled $W_{k}$ with $k>m$, and the letter may change when we cross an edge labeled $W_{l}$ for $l<m$. However, if the letter changes when we cross an edge labeled $W_{l}$, then it must change every time we cross such an edge. Therefore, by induction, there are an even number of changes at position place $\left(W_{m}\left(x_{i_{m}}\right)\right)$ caused by edges labeled $W_{l}$ with $l<m$ and none caused by edges labeled $W_{k}$ for $k>m$. Since the letter in place $\left(W_{m}\left(x_{i_{m}}\right)\right)$ must have its original value when we return to $\alpha$ after going around the cycle, there must be an even number of edges labeled by $W_{m}$.

By Lemma 3.8, any induced graph $G$ can be two-colored. That is, there is a map from the the unlabeled version of $G$ to $\{0,1\}$ such that no two nodes connected by an edge have the same value. We now state the main combinatorial lemma used to prove Theorem 3.1.

Lemma 3.9. Let $W_{1}\left(x_{i_{1}}\right), \ldots, W_{n}\left(x_{i_{n}}\right)$ be an admissible set of finite variable words such that for all $m \leq n,\left|W_{m}\right|<s$. Then, there is a two-coloring of $A^{s}$ such that for each $W_{m}\left(x_{i_{m}}\right)$ the following property holds. Let $k_{m}$ be such that $\left|W_{m}\left(x_{i_{m}}\right)\right|+k_{m}=s$. For each substitution instance $\widehat{W}_{m}\left(x_{i_{m}}\right)$ of all the variables in $W_{m}$ except $x_{i_{m}}$ and for all $\alpha \in A^{k_{m}}$

$$
c\left(\widehat{W}_{m}\left(a / x_{i_{m}}\right) * \alpha\right) \neq c\left(\widehat{W}_{m}\left(b / x_{i_{m}}\right) * \alpha\right) .
$$

Proof. Let $G$ be the graph induced on $A^{s}$ by the admissible set of finite variable words. If $\widehat{W}_{m}\left(x_{i_{m}}\right)$ and $\alpha$ are as in the statement of the lemma, then there is an edge labeled by $W_{m}$ from $\widehat{W}_{m}\left(a / x_{i_{m}}\right) * \alpha$ to $\widehat{W}_{m}\left(b / x_{i_{m}}\right) * \alpha$. Therefore, any two-coloring $c$ of $G$ has the desired property.

Lemma 3.10. Let $c: A^{<\mathbb{N}} \rightarrow\{0,1\}$ be a two-coloring and let $W$ be a finite ordered variable word in which $x_{0}, \ldots, x_{e}$ occur. Suppose there is a $k_{0} \in \mathbb{N}$ such that the following property holds. For each $k>k_{0}$, there exists $i_{k} \leq e$ such that for all substitution 
instances $\widehat{W}\left(x_{i_{k}}\right)$ of all variables in $W$ except $x_{i_{k}}$ and all $\alpha \in A^{k}$, we have

$$
c\left(\widehat{W}\left(a / x_{i_{k}}\right) * \alpha\right) \neq c\left(\widehat{W}\left(b / x_{i_{k}}\right) * \alpha\right) .
$$

Then, $W$ is not an initial segment of any homogeneous infinite ordered variable word $U$ in which all occurrences of $x_{0}, \ldots, x_{e}$ in $U$ occur in $W$.

Proof. Let $U$ be any infinite ordered variable word such that $W \subset U$ and all occurrences of $x_{0}, \ldots, x_{e}$ in $U$ occur in $W$. We need to show that $U$ is not homogeneous for the coloring $c$. Fix a variable $x_{m}$ such that $\operatorname{place}\left(U\left(x_{m}\right)\right)>|W|+k_{0}$. Set $k=\operatorname{place}\left(U\left(x_{m}\right)\right)-$ $|W|$ and note that $k>k_{0}$. Let $i_{k} \leq e$ be the value chosen for $k$ in the statement of the lemma.

Choose any substitution instance $\widehat{W}\left(x_{i_{k}}\right)$ of all the variables in $W$ except $x_{i_{k}}$. Choose a string $\alpha \in A^{k}$ such that $\widehat{W}\left(a / x_{i_{k}}\right) * \alpha$ and $\widehat{W}\left(b / x_{i_{k}}\right) * \alpha$ are both in $U(A)$ (if one of them is contained in $U(A)$, then the other must also be). By hypothesis,

$$
c\left(\widehat{W}\left(a / x_{i_{k}}\right) * \alpha\right) \neq c\left(\widehat{W}\left(b / x_{i_{k}}\right) * \alpha\right) .
$$

Therefore, $U$ is not homogeneous.

We can now prove Theorem 3.1.

Proof. We construct a computable coloring $c$ of $A^{<\mathbb{N}}$ such that no $\Delta_{2}^{0}$ infinite ordered variable word is homogeneous for $c$. Our construction proceeds in stages and at stage $s$ we color all strings in $A^{s}$. Because the construction is effective, our coloring is computable. We meet the requirements $\mathcal{R}_{e}$ : if $U_{e}(n)=\lim _{s} \varphi_{e}(n, s)$ is a total function which represents an infinite ordered variable word (under a fixed effective coding of $A \cup \operatorname{Var}$ ), then $U_{e}(A)$ is not homogeneous for $c$. Here, $\varphi_{e}$ is the standard effective list of all computable partial functions. By the Limit Lemma, if we meet all the $\mathcal{R}_{e}$ requirements, then we will have successfully diagonalized against all $\Delta_{2}^{0}$ infinite ordered variable words.

At stage $s$, we let $U_{e, s}$ be our approximation to $U_{e}$. We define $U_{e, s}$ to be the word formed by the longest initial segment of $\varphi_{e, s}(0, s), \ldots, \varphi_{e, s}(s-1, s)$ which converges. Notice that $\left|U_{e, s}\right| \leq s$ and that if $U_{e}$ is an infinite ordered variable word, then we will eventually see longer and longer initial segments of $U_{e, s}$ which are correct. At stage 0 we color the empty sequence arbitrarily and do nothing else.

\section{Construction at Stage $s$.}

For each $e \leq s$, check if $U_{e, s}$ is a finite variable word in which $x_{0}, \ldots, x_{e+1}$ all occur. If not, then we ignore $U_{e, s}$ at this stage. If so, then define $V_{e, s}$ to be the initial segment of $U_{e, s}$ which ends just before the first occurrence of $x_{e+1}$. If $V_{e, s}$ is a finite ordered variable word, then we say $V_{e, s}$ requires attention. Otherwise, we ignore $V_{e, s}$ at this stage.

Let $V_{j_{0}, s}, \ldots, V_{j_{n}, s}$ be the words requiring attention and assume that $j_{0}<j_{1}<\cdots<$ $j_{n}$. For each $m \leq n$, pick a pivot variable $x_{i_{m}}$ for $V_{j_{m}, s}$ such that $i_{m} \leq j_{m}$ and for any $m \neq p$,

$$
\operatorname{place}\left(V_{j_{m}, s}\left(x_{i_{m}}\right)\right) \neq \operatorname{place}\left(V_{j_{p}, s}\left(x_{i_{p}}\right)\right) .
$$

These choices can be made by starting with $V_{j_{0}, s}$ and proceeding to $V_{j_{n}, s}$ since each $V_{j_{m}, s}$ has $j_{m}+1$ variables occurring in it. 
Next, color the strings in $A^{s}$ such that the following property holds for each $V_{j_{m}, s}$. Let $k_{m}$ be such that $\left|V_{j_{m}, s}\right|+k_{m}=s$. For each substitution instance $\widehat{V}_{j_{m}, s}\left(x_{i_{m}}\right)$ of all the variables in $V_{j_{m}, s}$ except $x_{i_{m}}$ and for all $\alpha \in A^{k_{m}}$,

$$
c\left(\widehat{V}_{j_{m}, s}\left(a / x_{i_{m}}\right) * \alpha\right) \neq c\left(\widehat{V}_{j_{m}, s}\left(b / x_{i_{m}}\right) * \alpha\right) .
$$

The coloring can be defined consistent with these requirements by the Lemma 3.9. (If no words require attention at stage $s$, then color $A^{s}$ arbitrarily.) Proceed to stage $s+1$. End of Construction

It remains to check that we have met the requirements $\mathcal{R}_{e}$. Assume that $U_{e}$ is total and represents an infinite ordered variable word. Set $n=\operatorname{place}\left(U\left(x_{e+1}\right)\right)$ and fix a stage $t>n$ such that $U_{e, t}(i)=U_{e}(i)$ for all $i \leq n$. For every stage $s \geq t, V_{e, s}$ is defined and is equal to $U_{e} \mid n$. Therefore, $V_{e, s}$ has reached a limit which we call $V_{e}$. Furthermore, $V_{e}$ requires attention at every stage $s \geq t$. Let $k_{0}=t-\left|V_{e}\right|$. Consider any $k>k_{0}$, let $s=t+\left(k-k_{0}\right)$ and notice that $\left|V_{e}\right|+k=s$. At stage $s$ of our construction, we pick a pivot variable for $V_{e}$ from among $x_{0}, \ldots, x_{e}$. Denote this variable by $y_{s}$. Our coloring at stage $s$ guarantees that for all strings $\alpha \in A^{k}$ and all substitution instances $\widehat{V}_{e}\left(y_{s}\right)$ of all variables in $V_{e}$ except $y_{s}$, we have $c\left(\widehat{V}_{e}\left(a / y_{s}\right) * \alpha\right) \neq c\left(\widehat{V}_{e}\left(b / y_{s}\right) * \alpha\right)$. By Lemma 3.10 , we know that $V_{e}$ is not the initial segment of an infinite ordered variable word which is homogeneous for $c$. However, by assumption, $V_{e}$ is an initial segment of $U_{e}$ and therefore, we have met requirement $\mathcal{R}_{e}$.

\section{Open Problems}

Problem 1. In the previous section, we used a diagonalization argument to show that $\operatorname{OVW}(2,2)$ and $\operatorname{VW}(2,2)$ are not provable in $\mathrm{WKL}_{0}$. Can this be replaced with a coding argument which shows that, for at least some values of $k$ and $l$, OVW $(k, l)$ and $\mathrm{VW}(k, l)$ imply $\mathrm{ACA}_{0}$ ?

Problem 2. Determine the set theoretic strength required to prove $\operatorname{OVW}(k, l)$ and $\mathrm{VW}(k, l)$. The classical proof of $\mathrm{OVW}(k, l)$ given in Carlson and Simpson [1] goes beyond arithmetic comprehension. Can these principles be proved in $\mathrm{ACA}_{0}$ ?

Problem 3. Close the gap between $\Pi_{1}^{1}-\mathrm{CA}_{0} \vdash \operatorname{ODRT}(k, l)$ and $\operatorname{RCA}_{0}+\operatorname{ODRT}(k, l) \vdash$ $\mathrm{ACA}_{0}$.

There is another closely related infinite variable word principle which we will denote $\operatorname{VWI}(k, l)$. Given a finite alphabet $A$, we say that an $\mathbb{N}$-sequence $W$ of elements from $A \cup \operatorname{Var}$ is a variable word with infinitely occurring variables if each variable $x_{n}$ occurs at least once in $W$. Note that variables are allowed to occur infinitely often, but are not required to do so. The principle $\operatorname{VWI}(k, l)$ is the same as $\mathrm{VW}(k, l)$ except the homogeneous word is allowed to be a variable word with infinitely occurring variables. $\operatorname{VWI}(k, l)$ is clearly implied by both $\operatorname{OVW}(k, l)$ and $\operatorname{VW}(k, l)$, but our diagonalization techniques in Section 3 do not immediately work for $\operatorname{VWI}(2,2)$. $\mathrm{VW}(k, l)$ is of interest since it is the actual combinatorial principal required by Carlson and Simpson in their proof of the Dual Ramsey Theorem. 
Problem 4. Is $\operatorname{VWI}(k, l)$ proof theoretically simpler than $\mathrm{VW}(k, l)$ ? In particular, is it provable in $\mathrm{WKL}_{0}$ or even effectively true?

\section{REFERENCES}

[1] T.J. Carlson \& S.G. Simpson, A dual form of Ramsey's Theorem, Advances in Mathematics 53 (1984), 265-290.

[2] Y.L. Ershov, S.S. Goncharov, A. Nerode \& J.B. Remmel, Ed., "Handbook of Recursive Model Theory (Two Volumes)" Elsevier, Amsterdam 1998.

[3] H. Friedman \& S.G. Simpson, Issues and problems in reverse mathematics, in "Computability Theory and Its Applications: Current Trends and Open Problems" (P.A. Cholak, S. Lempp, M. Lerman \& R.A. Shore, Ed.) American Mathematical Society, Providence 2000, 127-144.

[4] C.G. Jockusch, Jr., Ramsey's theorem and recursion theory, Journal of Symbolic Logic 37 (1972), 268-280.

[5] F.P. Ramsey, On a problem of formal logic, Proceedings of the London Mathematical Society 30 (1930), 264-286.

[6] S.G. Simpson, Recursion theoretic aspects of the Dual Ramsey Theorem, in "Recursion Theory Week" (H.-D. Ebbinghaus, G.H. Müller \& G.E. Sacks, Ed.) Springer-Verlag, New York 1985, 356-371.

[7] S.G. Simpson, "Subsystems of second order arithmetic", Springer-Verlag, New York 1999.

[8] T.A. Slaman, A note on Dual Ramsey Theorem, 4 pages, unpublished, January 1997.

[9] R.I. Soare, "Recursively enumerable sets and degrees", Springer-Verlag, New York 1987.

[10] E. Specker, Ramsey's theorem does not hold in recursive set theory, in "Logic Colloquium '69 (Proc. Summer School and Colloq., Manchester, 1969)", North-Holland, Amsterdam 1971, 439442.

Joseph S. Miller, Department of Mathematics, Indiana University, Bloomington, INDIANA 47405-5701, USA

E-mail address: millerj7@indiana.edu

Reed Solomon, Department of Mathematics, University of Connecticut, Storrs, ConneCTicut 06269-3009, USA

E-mail address: solomon@math.uconn.edu 